\title{
Qualidade de vida em crianças
}

\section{e adolescentes com dermatite atópica e seus cuidadores}

\section{Quality of life in children and adolescents with atopic dermatitis and their caregivers}

Rev Port Imunoalergologia 202I; 29 (I):39-48

Priscilla Santos', Gabriela Dias², Saint Clair Junior³, Ana Maria Cerqueira'

' Hospital Municipal Jesus, Rio de Janeiro, Brasil

${ }^{2}$ Universidade do Estado do Rio de Janeiro, Rio de Janeiro, Brasil

${ }^{3}$ Instituto Fernandes Figueiras, Rio de Janeiro, Brasil

\section{RESUMO}

Introdução: A dermatite atópica (DA) é uma doença inflamatória crônica e a avaliação da qualidade de vida e da gravidade da doença é importante para auxiliar a abordagem clínica e a terapêutica. Objetivo: Avaliar o impacto na qualidade de vida da DA em crianças e adolescentes e seus cuidadores e correlacionar com a gravidade da doença. Materiais e métodos: Estudo transversal, com avaliação de características sócio-demográficas e aplicação de questionários nos cuidadores e pacientes pediátricos com DA consultados entre março a maio de 2018 , em ambulatório de dermatologia. A gravidade foi avaliada conforme o Scoring Atopic Dermatitis (SCORAD) e classificada como leve, moderada ou grave. O Dermatitis Family Impact Questionnaire (DFI) e o Children's Dermatology Life Quality Index (CDLQI) foram utilizados para avaliação da qualidade de vida. Resultados: Foram avaliados 34 pacientes com DA entre 4 e 15 anos. A média do SCORAD foi 29,14 (DP $\pm 14,85$ ) sendo $32 \%$ leve, $44 \%$ moderada e $24 \%$ grave. A média do CDLQI foi 7,32 (DP $\pm 5,91$ ) e do DFI de 12,62 (DP $\pm 7,34$ ). O domínio mais comprometido no DFI foi o impacto global na vida do cuidador, seguido pelas despesas com tratamento. Na avaliação do CDLQI, os itens com os piores índices foram o prurido e alteração do sono. Observou-se diferença significativa na qualidade de vida dos pacientes $(p=0,0 \mathrm{I})$ e cuidadores $(p=0,005)$ entre os pacientes com DA leve e grave, que não foi encontrada entre aqueles com DA leve e moderada e entre moderada e grave. Os índices de qualidade de vida dos cuidadores $(\rho=0,62 ; p<0,000 \mathrm{I})$ e dos 
pacientes $(\rho=0,53 ; p=0,00 I)$ mostraram uma correlação positiva e moderada com o SCORAD e entre si $(\rho=0,64$; $P<0,000 I)$. Conclusão: A DA interfere negativamente na qualidade de vida dos pacientes e de seus familiares, sendo os piores índices relacionados à maior gravidade da doença.

Palavras-chave: Adolescentes, crianças, dermatite atópica, qualidade de vida.

\section{ABSTRACT}

Introduction: Atopic dermatitis $(A D)$ is a chronic inflammatory disease and the assessment of the quality of life and the severity of the disease is important to help the clinical and therapeutic approach. Objective: To assess the impact of $A D$ on the quality of life of patients and caregivers and correlate with the severity of the disease. Material and Methods: Cross-sectional study, with assessment of socio-demographic characteristics and application of questionnaires in caregivers and pediatric patients with AD observed between March and May 2018, in a dermatology clinic. Severity was assessed according to the Scoring Atopic Dermatitis (SCORAD) and classified as mild, moderate or severe. The Dermatitis Family Impact Questionnaire (DFI) and the Children's Dermatology Life Quality Index (CDLQI) were used to assess quality of life. Results: Thirty-four patients with AD between 4 and 15 years old were evaluated. The SCORAD mean was 29.14 (SD \pm 14.85), 32\% mild, $44 \%$ moderate and $24 \%$ severe. The CDLQI mean was 7.32 (SD \pm 5.91 ) and the DFI was 12.62 (SD \pm 7.34 ). The most compromised domain in the DFI was the global impact on the caregiver's life, followed by treatment expenses. In the CDLQI evaluation, the items with the worst indexes were itching and sleep disorders. There was a significant difference in the quality of life of patients $(p=0.0 \mathrm{I})$ and caregivers $(p=0.005)$ between patients with mild and severe AD, which was not found between moderate and severe AD. The quality of life indexes of caregivers $(\rho=0.62 ; p<0.000 \mathrm{I})$ and patients $(\rho=0.53 ; p=0.00 \mathrm{I})$ showed a positive and moderate correlation with SCORAD and with each other $(\rho=0.64 ; p<0.0001)$. Conclusion: AD interferes negatively in the quality of life of patients and their families, with the worst rates related to the greater severity of the disease.

Keywords: Adolescents, atopic dermatitis, children, quality of life.

\section{INTRODUÇÃO}

A dermatite atópica (DA) é uma doença inflamatória crônica, de caráter genético, multifatorial, recidivante, de gravidade variável caracterizada por prurido intenso, podendo se apresentar em três fases clínicas diferentes: aguda (vesículas e crostas); subaguda (pápulas e placas secas, escamosas e eritematosas) e crônica (liquenificação e espessamento da pele). Pode causar alteração do sono, irritabilidade, estresse para o paciente e sua família. É a dermatite crônica mais comum na infância, e o diagnóstico é essencialmente clínicol,2.

A prevalência aumentou nas últimas três décadas, principalmente em países desenvolvidos, sendo acometidos em torno de $15-20 \%$ das crianças e $1-3 \%$ dos adultos no mundo inteiro ${ }^{1,2}$. Pode estar associada a outras manifestações alérgicas como rinite, asma e alergia alimentar².

A DA tem um impacto importante na qualidade de vida dos pacientes e de seus familiares, com consideráveis encargos pessoais, sociais e financeiros para a família e a 
comunidade $^{2,3}$. Frequentemente, o estresse pessoal e familiar não é valorizado porque a DA é considerada uma doença dermatológica simples e a avaliação médica concentra-se nos aspectos físicos da doença ${ }^{4}$. Dentre os fatores que contribuem para o estresse familiar, destacam-se a privação do sono, perda de emprego, tempo para o cuidado dos pacientes e custos financeiros ${ }^{3}$.

Os questionários do Children's Dermatology Life Quality Index (CDLQI) e o Dermatitis Family Impact Questionnaire (DFI) foram traduzidos em várias línguas e são usados para avaliação da qualidade de vida das crianças e dos familiares. São autoexplicativos e podem ser aplicados em várias dermatoses. Esses questionários abrangem vários domínios como sintomas e sentimentos, humor, gravidade da doença, alimentação, lazer, atividades domésticas, escola ou férias, relacionamentos pessoais, sono, gastos e tratamento ${ }^{5,6}$.

A avaliação da qualidade de vida e da gravidade da doença é importante para auxiliar a abordagem clínica e a terapêutica diária ${ }^{5}$. Assim, este estudo tem como objetivo avaliar o impacto na qualidade de vida (QV) da DA em pacientes e cuidadores, como também correlacionar com a gravidade da doença.

\section{MATERIAL E MÉTODOS}

O estudo foi transversal com aplicação de questionários nos cuidadores e pacientes pediátricos com DA, no período de março a maio de 2018 , em ambulatório de dermatologia. O diagnóstico de DA foi clínico e baseado nos critérios de Hanifin e Rajka ${ }^{7}$. Foram incluídos todos os cuidadores e pacientes entre 4 e 16 anos que foram consultados sucessivamente no período e concordaram em participar. Foram excluídos pacientes com outras doenças dermatológicas. $O$ estudo foi submetido ao Comitê de Ética em Pesquisa da instituição, recebendo aprovação.

Dados sociodemográficos como idade, gênero, cor, município de moradia, escolaridade dos pais, atopia fa- miliar, renda familiar, início da doença, tempo médio de doença, período de acompanhamento no serviço, internamento pela doença, uso de medicamentos e comorbidades associadas foram coletados durante as consultas em ficha elaborada para o estudo.

A gravidade da DA foi mensurada por meio do índice SCORAD (Scoring Atopic Dermatitis), que considera a extensão, gravidade da lesão e a presença de sintomas subjetivos, como prurido e perda de sono ${ }^{8}$. A doença foi classificada como leve (pontuação menor que 25), moderada (pontuação entre 25 e 50) ou grave (pontuação $>50)^{9}$.

Os questionários utilizados para avaliação da QV foram o Dermatitis Family Impact Questionnaire (DFI) e o Children`s Dermatology Life Quality Index (CDLQI) ${ }^{6}$, validados para o português brasileiro. $\mathrm{O}$ DFI avalia a interferência da DA na vida familiar, alterações emocionais, do sono, nas tarefas domésticas, na alimentação, nas atividades de lazer, no relacionamento conjugal e nos custos do tratamento. O CDLQI verifica o comprometimento da QV em diferentes aspectos: sintomas, sentimentos, lazer, escola, relações pessoais, sono e tratamento. Ambos são compostos por 10 questões referentes à última semana. Cada resposta recebe um escore de 0 a 3 pontos. Quanto maiores os valores desse escore, maior é o impacto da doença na QV, ou seja, pior é a qualidade de vida. $O$ valor máximo obtido é de 30 pontos. Foi avaliada a qualidade de vida e a gravidade da DA de acordo com as seguintes características: sexo, raça, escolaridade dos cuidadores, renda familiar, idade de início da doença, tempo médio de doença e uso de imunossupressor.

A análise estatística foi realizada usando o software de SPSS (Statistical Software for Social Sciences), versão 20.0. As estatísticas descritivas foram relatadas por frequência e média \pm desvio padrão (DP). As taxas de prevalência são mostradas como percentagens. $O$ teste qui-quadrado foi utilizado para estudar a relação entre variáveis qualitativas. Foram utilizados testes não paramétricos (Mann Whitney ou Kruskal Wallis) 
para estudar a relação entre variáveis contínuas. A comparação entre os índices de QV entre si e com os escores de gravidade da doença foi realizada através da correlação de Spearman. A significância foi alcançada com $\mathrm{p}<0,05$.

\section{RESULTADOS}

A amostra foi composta por $62 \%$ (2I) de crianças do gênero feminino, $56 \%$ (19) entre 10 e 15 anos e $44 \%$ (I5) entre 4 e 9 anos. A média de idades dos pacientes foi 9,75 anos (DP $\pm 3,00$ ) e dos pais 39,00 anos (DP $\pm 4,50$ ). A escolaridade dos pais foi em $44 \%$ (I5) ensino fundamental (que é uma das etapas de educação básica no Brasil, com duração de nove anos) e $41 \%$ (14) ensino médio (que é a parte do ensino secundário ministrado aos adolescentes até 19 anos). A maioria dos cuidadores $94 \%$ (32) eram mães. A maioria dos pacientes $64,7 \%$ (22) apresentou história positiva para atopia familiar. A média da renda familiar mensal foi de $R \$ 2.337,00$ ( $D P \pm 674,57$ ), equivalente a 537,24 euros (pela paridade do poder de compra em maio de 2018), com média de 4 pessoas por família, e 92\% (3I) residiam no Rio de Janeiro. Três responsáveis referiram ter deixado o emprego para cuidar dos filhos, pelas idas frequentes aos atendimentos médicos, tendo uma responsável referido ter trocado o emprego formal pelo informal (se tornou autônoma), pelo mesmo motivo.

Cerca de 26\% (9) dos pacientes apresentaram sintomas no primeiro ano de vida e $74 \%$ (25) apresentaram os sintomas entre 2 e 5 anos de idade. $O$ tempo médio de doença foi de 6,4 anos ( $D P \pm 3,03$ ), de diagnóstico da dermatite atópica foi de 5,8 anos ( $D P \pm 0,7$ ) e de acompanhamento no serviço foi de 17,88 meses (DP \pm 24,66 meses). Internação por dermatite atópica foi relatada por $41 \%$ (14) dos pacientes, sendo que 64\% (9) destes se internaram mais de uma vez pela doença, com média de 9 dias (7-30) de internamento. Todos os pacientes foram internados por infecção secundária com piora da gravidade da dermatite atópica, devido a baixas condições de higiene, cuidados precários com a pele e baixa adesão ao tratamento. Um paciente de II anos, masculino com DA grave, ficou internado por 30 dias por infecção secundária associada à depressão. Em torno de $53 \%$ (18) pacientes estavam em uso regular de anti-histamínico em doses padronizadas para a idade (13 pacientes em uso de hidroxizine, um em uso de dexclorfeniramida, dois em uso de cetirizina e outros dois em uso de loratadina - que são os anti-histamínicos disponibilizados gratuitamente pelo hospital), $9 \%$ (3) em uso de loratadina apenas quando apresentavam prurido, $11 \%$ (4) estavam em uso de imunossupressor (todos usando metotrexate que, apesar de ser off-label, é eficaz para a DA grave e disponibilizado pelo hospital gratuitamente para os pacientes, $18 \%(6)$ em uso de corticoesteroide oral durante o período, $9 \%$ (3) estavam em uso de corticoesteroides tópicos (por até 10 dias) e $9 \%$ (3) em uso tópico de inibidores de calcineurina (por até 14 dias).

As comorbidades mais prevalentes foram a rinite em 67\% (23) com sensibilização aos ácaros domésticos (Dermatophagoides pteronyssinus, Dermatophagoides farinae e Blomia tropicalis), asma em $38 \%$ (I3), obesidade em $20 \%$ (7) e alergia alimentar em II\% (4) dos pacientes (Quadro I). Três pacientes apresentaram alergia ao leite de vaca e um paciente, ao ovo. Apenas um responsável referiu que a alergia alimentar precedeu os sintomas da dermatite atópica, os outros não souberam precisar. Outros inalantes (epitélio de animais, pólens, fungos, etc.) não foram avaliados, por não estarem disponíveis no hospital, durante o período de avaliação.

A média do SCORAD foi 29,14 (DP $\pm 14,85$ ) o escore variou de 0 a 81 e, segundo a classificação, $32 \%$ (II) leve, $44 \%$ (15) moderada e $24 \%$ (8) grave. A média do CDLQI foi 7,32 (DP $\pm 5,91$ ) e do DFI de 12,62 (DP $\pm 7,34$ ). A piora da qualidade de vida dos familiares (DFI) foi relacionada ao efeito, na vida do principal responsável, provocado pela ajuda com o tratamento dos pacientes, seguido pelas despesas com a doença; enquanto o menor 
Quadro I. Características socioeconômicas e clínicas da amostra

\begin{tabular}{|c|c|}
\hline Variáveis & $n=34$ \\
\hline \multicolumn{2}{|l|}{ Sexo n (\%) } \\
\hline Masculino & $13(38)$ \\
\hline Feminino & $21(62)$ \\
\hline \multicolumn{2}{|l|}{ Idade dos pacientes $\mathbf{n}(\%)$} \\
\hline 4-9 anos & $15(44)$ \\
\hline $10-15$ anos & $19(56)$ \\
\hline Média (DP) & $9.75(3.00)$ \\
\hline \multicolumn{2}{|l|}{ Idade dos cuidadores } \\
\hline Média (DP) & $39(4.50)$ \\
\hline \multicolumn{2}{|l|}{ Escolaridade dos cuidadores n (\%) } \\
\hline Ensino fundamental & $15(44)$ \\
\hline Ensino médio & $14(4 I)$ \\
\hline Ensino superior & $5(15)$ \\
\hline $\begin{array}{c}\text { Renda familiar } \\
\text { Média (DP) }\end{array}$ & $\begin{array}{c}\mathrm{R} \$ 2.337,00 \\
(674,57)\end{array}$ \\
\hline \multicolumn{2}{|l|}{ Idade de início dos sintomas } \\
\hline$<$ I ano & $9(26)$ \\
\hline 2 a 5 anos & $25(74)$ \\
\hline \multicolumn{2}{|l|}{ Tempo de doença (anos) } \\
\hline Média (DP) & $6,4(3.03)$ \\
\hline \multicolumn{2}{|l|}{ Tempo de diagnóstico (anos) } \\
\hline Média (DP) & $5.8(0.70)$ \\
\hline \multicolumn{2}{|l|}{ Tempo de acompanhamento (meses) } \\
\hline Média (DP) & $17,88(24.66)$ \\
\hline \multicolumn{2}{|l|}{ Comorbidades (\%) } \\
\hline Rinite alérgica & $23(67)$ \\
\hline Asma brônquica & $13(38)$ \\
\hline Obesidade & $7(20)$ \\
\hline Alergia alimentar & $4(11)$ \\
\hline \multicolumn{2}{|l|}{ Tratamento medicamentoso $\mathrm{n}(\%)$} \\
\hline Anti-histamínico oral contínuo & $18(53)$ \\
\hline Anti-histamínico oral sob demanda & $3(9)$ \\
\hline Imunossupressor (metotrexato) & $4(\mathrm{II})$ \\
\hline Corticosteroide oral (curso curto) & $6(18)$ \\
\hline Corticosteroide tópico (curso curto) & $3(9)$ \\
\hline Inibidor da calcineurina (curso curto) & $3(9)$ \\
\hline
\end{tabular}

impacto foi relacionado à preparação da comida/alimentação e ao relacionamento entre os familiares (Quadro 2). Nas crianças/adolescentes, os piores índices na qualidade de vida (CDLQI) foram causados pelo prurido
Quadro 2. Questionário Dermatitis Family Impact Questionnaire (DFI)

\begin{tabular}{|c|c|}
\hline Domínios $(n=34)$ & Média (DP) \\
\hline Interferência nas tarefas domésticas & $1.23(1.00)$ \\
\hline Interferência no preparo das refeições & \\
\hline e alimentação & $1.00(1.08)$ \\
\hline Perturbação no sono & I.I7 (1.17) \\
\hline Interferência nas atividades de lazer & $1.11(1.05)$ \\
\hline Tempo gasto em compras & I.II (I.20) \\
\hline Gastos com o tratamento & $1.85(0.87)$ \\
\hline Sentimentos de cansaço e exaustão & $1.61(1.02)$ \\
\hline Perturbação emocional & $1.05(1.10)$ \\
\hline Interferência no relacionamento conjugal & $0.73(1.06)$ \\
\hline Interferência global na vida do principal cuidador & $1.91(0.98)$ \\
\hline Escore total & $12.73(7.10)$ \\
\hline
\end{tabular}

Escore do DFI: mínimo:0, máximo: 3 por questão

Quadro 3. Questionário Children`s Dermatology Life Quality Index (CDLQI)

\begin{tabular}{|l|c|}
\hline \multicolumn{1}{|c|}{ Domínios $(\mathrm{n=34)}$} & Média (DP) \\
\hline Prurido & $\mathrm{I} .60(\mathbf{0 . 8 )}$ \\
Constrangimento & $0.97(0.92)$ \\
Interferência nas amizades & $0.29(0.24)$ \\
Interferência no vestuário & $0.79(0.86)$ \\
Interferência nas atividades de lazer & $0.47(0.84)$ \\
Interferência nas atividades esportivas & $0.76(1.08)$ \\
Interferência nas atividades escolares e férias & $0.5(0.81)$ \\
Bullying & $0.32(0.75)$ \\
Interferência no sono & $\mathbf{I . 0 2 ( 1 . 0 9 )}$ \\
Perturbação causada pelo tratamento & $0.76(0.90)$ \\
Escore total & $7.47(5.75)$ \\
\hline
\end{tabular}

Escore do CDLQI: mínimo: 0, máximo: 3 por questão

(domínio sintomas e sentimentos) e alteração de sono (domínio sono); os índices de menores pontuações foram os relacionados ao relacionamento com amigos e bullying (domínio relações pessoais) (Quadro 3).

Observou-se diferença significativa na qualidade de vida dos pacientes $(P=0,01)$ e cuidadores $(p=0,005)$ entre os pacientes com DA leve e grave que não foi encontrada entre aqueles com DA leve e moderada, nem entre moderada e grave (Figuras I e 2). A avaliação da qualidade de vida de acordo com a idade dos pacientes, gênero, escolaridade dos cuidadores e tempo de 


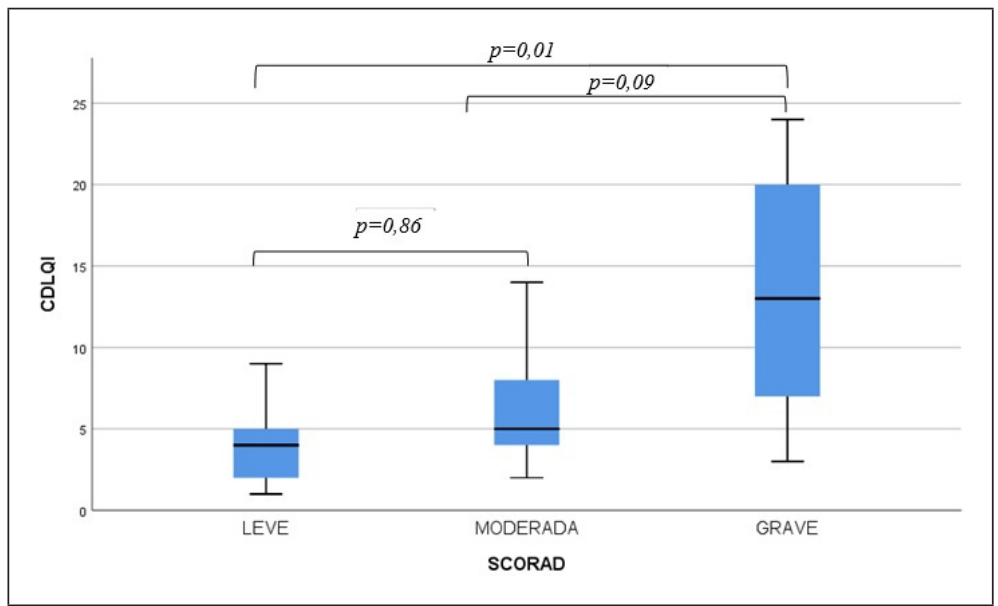

CDLQI: Children's Dermatology Life Quality Index; SCORAD: Scoring Atopic Dermatitis.

Figura I. Relação entre gravidade da dermatite atópica e índices de qualidade de vida dos pacientes

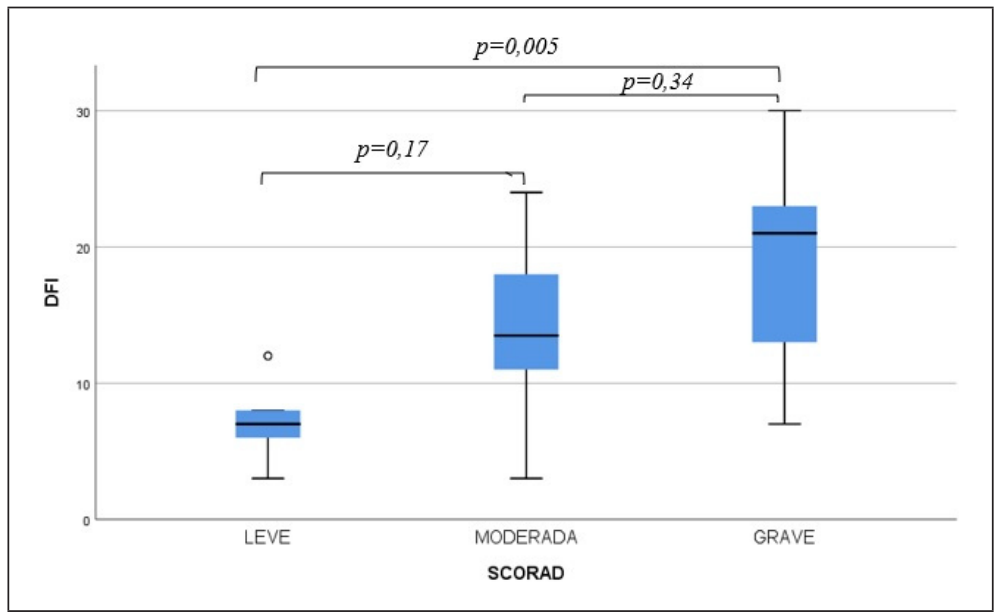

DFI: Dermatitis Family Impact/Questionnaire; SCORAD: Scoring Atopic Dermatitis

Figura 2. Relação entre gravidade da dermatite atópica e índices de qualidade de vida dos cuidadores

acompanhamento no serviço não demonstrou diferença estatística relevante. Os índices de qualidade de vida dos cuidadores $(\rho=0,62 ; p<0,000 I)$ e dos pacientes $(\rho=0,53 ; p=0,00 I)$ mostraram uma correlação positiva e moderada com o SCORAD e entre si $(\rho=0,64$; $\mathrm{P}<0,000 \mathrm{I})$.

\section{DISCUSSÃO}

A DA é mais prevalente na infância; $50 \%$ desses pacientes desenvolvem outros sintomas alérgicos no primeiro ano de vida (2). Aproximadamente $60 \%$ dos pacientes desenvolvem DA no primeiro ano de vida, e $90 \%$ 
nos primeiros 5 anos (I). Na casuística apresentada, 26\% iniciaram os sintomas no primeiro ano de vida (menos que o relatado na literatura), e $74 \%$ apresentaram início dos sintomas entre dois e cinco anos. A atopia familiar foi observada em aproximadamente $65 \%$ dos pacientes, o que é um fator de risco para o desenvolvimento da DA'. Do total, $67 \%$ apresentava rinite, $38 \%$ asma e $11 \%$ alergia alimentar. De acordo com a literatura, aproximadamente um terço dos pacientes com DA desenvolve asma, e dois terços desenvolvem rinite alérgica'. Sabe-se que a DA pode estar associada à alergia alimentar, embora não existam dados recentes sobre essa proporção!.

A DA representa um fardo significativo para os recursos de assistência à saúde e qualidade de vida dos pacientes. Os fatores que contribuem para o estresse familiar incluem privação do sono, perda de emprego, tempo necessário para cuidar da dermatite atópica e custos financeiros ${ }^{2,3}$, como observado nas respostas dos questionários. Os custos financeiros para a família e a comunidade incluem custos diretos médicos e hospitalares dos tratamentos, além de custos indiretos decorrentes da perda de emprego. Na população estudada, $41 \%$ dos pacientes apresentaram ao menos uma internação, - que aumenta os custos com a doença e interfere negativamente na qualidade de vida da criança/adolescente e dos cuidadores. Esse alto índice de internamento pode ser provavelmente pelas baixas condições de higiene, poucos cuidados com a saúde, condições de moradia ruins, controle ambiental inadequado e baixo poder econômico dessa população.

$\mathrm{Na}$ qualidade de vida dos pacientes, os fatores mais prevalentes foram o prurido e o impacto no sono, enquanto dos pais foi o efeito provocado pela ajuda com o tratamento dos pacientes, seguido pelas despesas com a doença. Os gastos com o tratamento são altos, pois são necessários sabonetes, hidratantes, medicamentos, transporte para a realização das consultas e dos exames complementares (quando necessários), entre outros. Esse efeito nos custos da doença pode ser explicado pelas características socioeconômicas e regionais presentes em nossa amostra, visto que a maioria reside em comunidades e é atendida no Sistema Único de Saúde. Os custos da DA são elevados e comparáveis a doenças como psoríase e asma, estimados em cerca de um bilhão anual nos EUA ${ }^{10}$.

Políticas públicas são importantes na implementação da diminuição dos custos de doenças crônicas, tendo o médico generalista e o médico de família um papel fundamental. Em Portugal, existe a Rede Médico-Sentinela que funciona como um sistema de vigilância de doenças crônicas". No Brasil, os médicos de família podem auxiliar a comunidade com os cuidados básicos e preventivos de saúde. Nos hospitais e em postos de saúde, poderia ser instituída uma sala de espera para os pacientes com doenças crônicas e seus familiares, antes dos atendimentos médicos. Haveria um programa de educação terapêutica multidisciplinar, com o auxílio de outros profissionais de saúde como enfermeiros, psicólogos, fisioterapeutas, nutricionistas e assistentes sociais com o objetivo de orientações sobre a doença, dando responsabilidade aos pacientes e aos seus responsáveis para contribuírem com as medidas preventivas e o tratamento, aumentando assim o vínculo com a equipe, a adesão e meIhorando o controle da DA.

Em um estudo chinês, os três itens no questionário CDLQI com pontuações mais altas foram o prurido, problemas de humor e distúrbios no sono nos pacientes com DA. Distúrbios no sono, fadiga e problemas de humor foram os três itens do questionário do DFI com maior pontuação ${ }^{12}$.

Em 2009, um estudo brasileiro realizado por Alvarenga TM e cols. mostrou em relação à pontuação do questionário DFI, maior impacto para os domínios que avaliam despesas com o tratamento, assim como foi evidenciado na nossa população, além do efeito sobre as compras da família, também a alteração do sono em outros membros da família e a dermatite atópica como causa de cansaço e exaustão para os familiares ${ }^{13}$.

Em 2017, Carvalho e cols. demonstraram a média do índice de QV da família de 9,I ( \pm 6,7), sendo os gastos com o tratamento, sentimento de exaustão e prejuízos 
no sono, os domínios mais frequentemente afetados ${ }^{14}$. Alvarenga e Caldeira, também no Brasil, descreveram índices menores $(8,5 \pm 4,4)^{13}$. Foram observados índices maiores em nosso estudo com DFI de 12,62 (DP $\pm 7,34$ ). Quando comparamos com o estudo realizado de 2009, podemos explicar os piores índices encontrados na nossa população por ser composta na maioria por pacientes com DA moderada/grave, enquanto na outra amostra a maioria era composta por DA leve. Mas isso não explica os dados encontrados em 2017, pois o SCORAD foi semelhante ao encontrado na nossa população.

Nesse mesmo estudo brasileiro realizado por CarvaIho e cols., o SCORAD variou de 0 a 77,8, sendo que $31,7 \%$ dos pacientes apresentavam DA leve, 36,6\% DA moderada e $31,7 \%$ DA grave ${ }^{14}$, dados semelhantes aos encontrados em nosso estudo. A gravidade da DA varia conforme a região. No entanto, a proporção de doentes graves é menor que moderados e leves ${ }^{15}$. Um estudo realizado com 78.300 pacientes adultos com DA em Portugal (0,7-1,6\% da população adulta portuguesa) atendidos em 20I7, demonstrou que 40-45\% tinham doença moderada ou grave, confirmando que a DA representa uma pequena proporção da população geral de $\mathrm{DA}^{15}$. Das 22354 crianças portuguesas menores que 16 anos, 3214 (I4,4\%) tinham DA leve.

Em outro estudo nacional realizado em Portugal em 2018, Carvalho D. et al. relataram, em uma amostra de 162 pacientes (adultos e crianças), que $87 \%$ dos pacientes estavam com DA moderada a grave, de acordo com o PO-SCORAD (4I,8\% moderado e $45,5 \%$ grave, respectivamente $)^{16}$. Apesar de terem usados outros questionários para a qualidade de vida dos pacientes nesse estudo, foi demonstrado que a DA tem um forte impacto negativo na qualidade de vida, e "sintomas" é o domínio com maior impacto, tanto para adultos como para crianças $^{16}$.

Em 2015, no Pará, foi realizado um estudo que mostrou a pontuação média sendo de 5,4 \pm 5, I para CDLQI, 6,6 $\pm 4,5$ para DFI e $28,3 \pm 19,8$ para SCORAD ${ }^{17}, 55 \%$ meninas, idade média $9,5 \pm 3,2$, sendo $51 \%$ entre 5 a 9 anos. Os índices do CDLQI e do DFI também foram menores, quando comparados a nossa amostra, mas o SCORAD, a média de idade, a prevalência pelo gênero feminino foram semelhantes. $\mathrm{Na}$ amostra estudada, a maioria era composta por crianças entre 10 e 15 anos (56\%); enquanto, no Pará, a maioria era entre 5 a 9 anos (5I\%).

A maioria das famílias ganha menos que $R \$ 2.500,00$ por mês, e $68 \%$ dos pacientes apresentam DA moderada/ grave com maior impacto nos custos. A maioria dos cuidadores era composta por mães $94 \%$, sendo que $59 \%$ eram donas de casa, para ficarem mais disponíveis ao tratamento dos filhos. Um estudo australiano avaliou o impacto nas famílias de cuidadores de crianças com DA e demonstrou que cuidar de uma criança com DA moderada ou grave foi significativamente mais estressante do que cuidar de uma criança com diabetes mellitus tipo $1^{18}$. O tempo gasto por dia no tratamento de crianças com DA era de 1,5 a 3 horas nesse estudo ${ }^{18}$. A sobrecarga causada por cuidar desses pacientes gera conflitos entre os pais e entre os outros filhos do casal, alterando a estrutura familiar ${ }^{13}$.

Em um estudo polonês, a qualidade de vida das mães foi mais afetada, quando comparada a dos pais. Na Polônia, assim como no Brasil, em muitas famílias, a mãe fica responsável pelos cuidados com os filhos e com a casa, dispensando mais tempo nos cuidados com a doença, interferindo negativamente nas suas atividades diárias ${ }^{19}$.

A alteração do sono pode afetar tanto as crianças e adolescentes, como os seus cuidadores. Fennessy M e cols. relataram que pacientes com dermatite atópica grave apresentaram uma média de 2,I horas perdidas por noite, enquanto a estimativa de perda de sono dos pais foi de 1,9 horas por noite ${ }^{4}$. As crianças com DA demonstram com mais frequência cansaço e irritabilidade durante o dia, considerados relacionados à falta de sono ${ }^{20}$.

Quanto maior a gravidade da DA, pior a qualidade de vida dos pacientes $(p=0,01)$ e dos cuidadores $(p=0,005)$. Kim e col. realizaram um estudo semelhante na Coréia, em 20I2, comprovando a correlação entre a gravidade da doença e o impacto dessa dermatose sobre qualidade de vida dos familiares e pacientes ${ }^{5}$. No Brasil, Alvarenga 
e col. em 2009 demonstraram que a DA interferiu negativamente na qualidade de vida dos pacientes pediátricos e dos familiares ${ }^{13}$.

Em concordância com o estudo realizado no Parál4, em 2015, nosso estudo demonstrou a correspondência entre a gravidade da doença (SCORAD) e a qualidade de vida do paciente (CDLQI) e do respectivo responsável (DFI). Quanto maior o SCORAD, menor a qualidade de vida da família ${ }^{21}$.

É importante identificar os pacientes com menor qualidade de vida, podendo auxiliar na prevenção dos sintomas e doenças psiquiátricas, pois mostra como a pessoa enfrenta $\circ$ fator estressante neste contexto ${ }^{6}$.

Os pacientes com DA e seus familiares devem ter uma abordagem multidisciplinar, a fim de melhorar a qualidade de vida, além de auxiliar na prevenção das exacerbações da doença e conscientizar os cuidadores e pacientes sobre os cuidados com a saúde.

Uma limitação do estudo realizado foi uma amostra de conveniência, uma vez que foram selecionados pacientes do ambulatório de dermatologia, em sua maioria, casos moderados/graves. Outra restrição do estudo, foi a avaliação de apenas pacientes do SUS (Sistema Único de Saúde), ou seja, pacientes com níveis socioeconômicos superiores não foram inseridos no estudo.

\section{CONCLUSÃO}

A DA interfere negativamente na qualidade de vida dos pacientes e de seus familiares, sendo os piores índices relacionados à maior gravidade da doença. $A$ interferência global da DA na vida dos cuidadores apresentou os piores índices; enquanto, nas crianças e adolescentes, o prurido foi o mais significativo.

O uso dos questionários de qualidade de vida dos pacientes e de seus cuidadores é um recurso que deve ser usado pelos médicos durante as consultas, para avaliar o perfil da população atendida, com o objetivo de um tratamento individualizado e novas estratégias terapêuticas.

\section{Conflito de interesses}

Os autores declaram que não existem conflitos de interesses.

\section{Contacto:}

Rua da Assembléia, 10 - sala 3407

Centro - Rio de Janeiro

Cep: 200II-90I

E-mail: pri.80@globo.com

\section{REFERÊNCIAS}

I. Avena-Woods C. Overview of atopic dermatitis. Am J Manag Care 2017;23(8 Suppl): SII5-SI23.

2. Nutten S. Atopic dermatitis: global epidemiology and risk factors. Ann Nutr Metab 2015;66(Suppl I):8-16.

3. Kemp, A. S. Cost of Illness of Atopic Dermatitis in Children. PharmacoEconomics 2003;21:105-13.

4. Fennessy M, Coupland S, PopayJ, Naysmith K. The epidemiology and experience of atopic eczema during childhood: a discussion paper on the implications of current knowledge for health care, public health policy and research. J Epidemiol Community Health 2000;54:58I-9.

5. Kim DH, Li K, Seo SJ, Jo SJ, Yim HW, Kim CM et al. Quality of life and disease severity are correlated in patients with atopic dermatitis. J Korean Med Sci 2012;27:1327-32.

6. C. Prati, C. Comparin, J. Catucci Boza, T. Ferreira Cestari. Validação para o português falado no Brasil do instrumento Escore da Qualidade de Vida na Dermatologia Infantil (CDLQI). Med Cutan Iber Lat Am 2010;38:229-33.

7. Hanifin JM, Rajka G. Diagnostic features of atopic dermatitis. Acta Derm Venereol (Suppl.) 1980; 92:44-7.

8. Severity scoring of atopic dermatitis: the SCORAD index. Consensus Report of the European Task Force on Atopic Dermatitis. Dermatology 1997;195:10-9.

9. Antunes AA, Solé D, Carvalho VO, Bau AEK, Kuschnir FC, Mallozi MC et. al. Guia prático de atualização em dermatite atópica - Parte I: etiopatogenia, clínica e diagnóstico. Posicionamento conjunto da Associação Brasileira de Alergia e Imunologia e da Sociedade Brasileira de Pediatria. Arq Asma Alerg Imunol 2017; Vol. I. N. ${ }^{\circ} 2$; I3I-57.

10. Mancini AJ, Kaulback K, Chamlin SL. The socioeconomic impact of atopic dermatitis in the United States: a systematic review. Pediatr Dermatol 2008;25:I-6.

II. Rodrigues AP, Fonseca RC, Matias-Dias C. Rede Médicos-Sentinela como Instrumento de Vigilância em Saúde [Pública]. Acta Med Port 2016;29:5-9.

12. Cheng Y, Zhang Z, Liu XY, He H, Chen J. Zhongguo Dang Dai Er Ke Za Zhi. Evaluation of Quality of Life of Children With Atopic 
Dermatitis Before and After Treatment. Chinise J Contemp Pediatrics 2017;19:682-7.

13. Alvarenga TM, Caldeira AP. Quality of life in pediatric patients with atopic dermatitis. J Pediatr (Rio J). 2009;85:415-20.

14. Carvalho SLC, Boguchewski AP, Nascimento FLS, Dalmas LM, Carvalho VO. Impacto da dermatite atópica na qualidade de vida da família. Braz J Allergy Immunol 20I7; I:305-I0.

15. Torres T, Ferreira EO, Gonçalo M, Mendes-Bastos P, Selores M, Filipe P. Update on Atopic Dermatitis. Acta Med Port 2019;32: 606-13.

16. Carvalho D, Aguiar P, Mendes-Bastos P, Palma-Carlos A, Freitas J, Ferrinho P. Quality of Life and characterization of patients with atopic dermatitis in Portugal - QUADEP study. J Investig Allergol Clin Immunol. 2020;30:430-8.
17. Bezerra ALC, Araújo FM, Santos MAL, Santos AAS, Pires Carla AA. Impact of atopic dermatitis on the quality of life of pediatric patients and their guardians. Rev Paul Pediatr 2017;35:5-10.

18. Su JC, Kemp AS, Varigos GA, Nolam TP. Atopic eczema: its impact on the family and financial cost. Arch Dis Child 1997;76:159-62.

19. Marciniak J, Reich A, Szepietowski JC. Quality of Life of Parents of Children with Atopic Dermatitis. Acta Derm Venereol 2017; 97:7II-4.

20. Dahl RE, Bernhisel-Broadbent J, Scanlon-Holdford S, Sampson HA, Lupo M. Sleep disturbances in children with atopic dermatitis. Arch Pediatr Adolesc Med 1995;149: 856-60.

21- Ben-Gashir, MA, Seed PT, Hay RJ. A qualidade de vida familiar e a gravidade da doença estão relacionadas à dermatite atópica infantil? J Eur Acad Dermatol Venereol 2002;16:455-62. 